\title{
Der Abschluss des Schengener Abkommens und dessen Umsetzung
}

Anfang der 1970er Jahre bestand also zwischen den EWG-Staaten eine weitgehende Freizügigkeit für Arbeitskräfte und deren Familienangehörige, einen Visumzwang gab es nicht mehr, Grenzkontrollen allerdings schon. Mit den zahlreichen Anwerbeabkommen hatte sich die grenzüberschreitende Arbeitsmigration zwischen den Mittelmeeranrainerstaaten und den Industrieländern in West-, Mittel- und Nordeuropa erheblich verstärkt. Ihre Folgen firmieren weiterhin häufig unter dem in vielerlei Hinsicht unzutreffenden Begriff der „Gastarbeiter“Migration. Die EWG-Staaten, sieht man von Italien ab, bildeten die wichtigsten Ziele der Arbeitsmigration im Rahmen der Anwerbeabkommen. Zwischen 1970 und 1974 kam allerdings mit den Anwerbestopp-Maßnahmen das Ende des Instruments der Anwerbeabkommen, das für mehr als fünfzig Jahre eine ausgesprochen hohe Bedeutung für die Migrationssituation in Europa gehabt hatte (hierzu und zum Folgenden: Berlinghoff 2013).

Das Ende der Anwerbung und die scharfe Begrenzung der Zuwanderung Anfang der 1970er Jahre bildeten das Ergebnis der seit den späten 1960er Jahren laufenden Debatten um die Kosten der Niederlassung von Arbeitskräften aus dem Ausland. Sie waren lange als nur temporär anwesend betrachtet worden, aber die Dauer ihres Aufenthalts wuchs, ebenso der Umfang des Familiennachzugs, auch die Zahl der Schülerinnen und Schüler aus dem Ausland stieg erheblich an. In den späten 1960er Jahren wurden die Folgen dieser Tendenz zur Niederlassung für Schulen, Kindergärten, das Sozialversicherungssystem, das Angebot auf dem freien Wohnungsmarkt und die Wahrung der Identität einer als homogen vorgestellten Nation als derart weitreichend eingeschätzt, dass eine weitere Anwerbung nicht mehr akzeptabel schien.

Den Anfang einer erheblichen Beschränkung der Zuwanderung machte die Schweiz 1970: Neuzuwanderungen wurden nur noch in dem Umfang zugelassen, in dem andere Ausländerinnen und Ausländer aus der Schweiz abgewandert 
waren, oder wenn ihnen eine Genehmigung zur unbefristeten Niederlassung erteilt worden war. 1971 beschloss die britische Regierung, dass nur noch jene Commonwealth-Bürgerinnen und -Bürger ungehindert nach Großbritannien einreisen durften, die nachweisen konnten, dass ihre Eltern oder Großeltern in Großbritannien geboren worden waren. Diese Regelung trat mit dem Beitritt Großbritanniens zur EWG am 1. Januar 1973 in Kraft und war eine Vorbedingung für die Aufnahme, denn die anderen EWG-Mitgliedstaaten wollten die freie Arbeitsaufnahme nicht-europäischer „British Subjects“ in ihren Ländern ausschließen (Layton-Henry 1992). 1972 folgten Beschränkungen weiterer Staaten, jetzt in der Form des Stopps der Anwerbung ausländischer Arbeitsmigrantinnen und Arbeitsmigranten: Schweden und Dänemark ließen nur noch Arbeitskräfte aus anderen skandinavischen Staaten zu. 1973 beendete nicht nur die Bundesrepublik Deutschland die Anwerbung ausländischer Arbeitskräfte, auch die Niederlande und Belgien blockierten die Arbeitsmigration von außerhalb der EWG. Den Abschluss bildete im Sommer 1974 der Anwerbestopp in Frankreich.

Die Maßnahmen beruhten zwar auf nationalen Entscheidungen und resultierten aus einer je spezifischen nationalen Debatte über die Folgen der Einwanderung. Dass die Anwerbestoppmaßnahmen in den Zielländern der Arbeitsmigration aber in relativ kurzer Frist aufeinanderfolgten, war auch einer zunehmenden Europäisierung der Diskussion geschuldet: Medien, Politik und Administration blickten sehr bewusst auf die Debatten über Migration und Niederlassung in anderen Ländern Europas. Darüber hinaus gab es im Prozess der europäischen Integration auf verschiedenen - zwischenstaatlichen und supranationalen - Ebenen immer häufiger genutzte Möglichkeiten des politischen und administrativen Austauschs über die jeweiligen Maßnahmen zur Bewältigung der als Gefahr wahrgenommenen Niederlassung von Arbeitskräften aus dem Ausland.

Der Stopp der Anwerbung bildete die Voraussetzung dafür, dass sich die Diskussion um eine Gemeinschaft ohne Binnengrenzen intensivierte. 1974 vereinbarten die Staats- und Regierungschefs der EWG-Länder auf dem Pariser Gipfel eine Passunion. Eine darüber erreichte Öffnung der Binnengrenzen leiste einen weitreichenden Beitrag zur Förderung einer europäischen Identität und forciere so die Integrationsbemühungen. Grenzkontrollen verursachten außerdem hohe Kosten für Staat und Wirtschaft, insbesondere international agierende Unternehmen seien im Nachteil. Eine Umsetzung des Vorhabens aber gelang nicht: Sorge um die nationale Sicherheit und Ängste vor einem Verlust migrationspolitischer Kontrolle der Mitgliedstaaten verhinderten sie. Als problematisch galt auch, dass mit einem Wegfall der Identitätskontrolle bei der Überquerung von Staatsgrenzen nicht nur Bürgerinnen und Bürger der EWG-Länder, sondern auch dort lebende Angehörige von Drittstaaten die Möglichkeit erhalten hätten, sich innerhalb der 
EWG ungehindert zu bewegen (Gehring 1998, S. 47 f.). Eine Passunion und eine Verringerung der Grenzkontrollen zwischen den Mitgliedstaaten erschien erst dann möglich, wenn ein Einheitspass für alle Bürgerinnen und Bürger der EWG-Staaten eingeführt worden sei. Außerdem müssten die ausländerrechtlichen Bestimmungen in den Mitgliedstaaten aufeinander abgestimmt werden (hierzu und zum Folgenden: Pudlat 2013; Siebold 2013).

Der Beschluss über die Einführung eines einheitlichen europäischen Passes erfolgte 1981, also mit siebenjähriger Verzögerung und just dann, als vor dem Hintergrund des Anstiegs der Zahl der Asylsuchenden in vielen EWG-Staaten die Öffnung der Binnengrenzen erneut als nicht opportun galt. Grundsätzlicher Widerstand kam zudem aus Großbritannien. Die britische Regierung wollte die europäische Integration auf das Feld der Ökonomie beschränkt sehen. Weil die Bevölkerung im Vereinigten Königreich in der Regel nicht über Personalausweise verfügte und keine Passpflicht bestand, gab es keine Personenkontrollen im Innern, sondern nur an den Außengrenzen. In einem grenzfreien Europa hätte Großbritannien entweder auf alle Personenkontrollen verzichten oder das Pass- und Kontrollsystem umbauen müssen. Dazu war die Regierung nicht bereit. Frankreich und die Bundesrepublik suchten deshalb einen Weg zur Aufhebung innereuropäischer Grenzkontrollen außerhalb des Rahmens der EWG.

Bundeskanzler Helmut Kohl und der französische Staatspräsident François Mitterrand vereinbarten 1984 im „Saarbrücker Abkommen“ einen Abbau der Kontrollen an der gemeinsamen Grenze in drei Schritten. Zunächst sollten nur noch Sichtkontrollen an den Grenzen durchgeführt werden, Fahrzeuge aber nicht mehr anhalten müssen. Vereinbart wurden zudem zoll- und devisenrechtliche Angleichungen sowie vermehrte Aktivitäten zur Eindämmung grenzüberschreitender Kriminalität und zur Verhinderung unerlaubter Einreisen an den Außengrenzen. Im zweiten Schritt sollte die Verlagerung der Grenzkontrollen an die Außengrenzen und der völlige Abbau der Binnengrenzen vorbereitet werden. Zur Umsetzung des dritten Schrittes räumten die beiden Staaten sich eine Frist bis Ende 1986 ein: Bis dahin seien die Mehrwert- und Verbrauchssteuersätze sowie die Vorschriften im Ausländer-, Betäubungsmittel- und Waffenrecht anzugleichen.

Dieses Maßnahmenpaket erwies sich als attraktiv für weitere EWG-Staaten. Sie bekundeten sogleich Interesse an dem Abschluss eines ähnlichen bilateralen Abkommens oder schlugen, wie Belgien, Luxemburg und die Niederlande, vor, die im Saarbrücker Abkommen getroffenen Regelungen multilateral auf die gemeinsamen Grenzen auszuweiten. Das war der Anstoß für die Verhandlungen zum im Juli 1985 im luxemburgischen Ort Schengen abgeschlossenen „Übereinkommen“ zwischen Belgien, der Bundesrepublik Deutschland, Frankreich, Luxemburg und den Niederlanden „betreffend den schrittweisen Abbau 
der Kontrollen an den gemeinsamen Grenzen“. Von den Erleichterungen beim Grenzübertritt, die die Regelungen des „Schengener Übereinkommens“, einem völkerrechtlichen Vertrag außerhalb des Rahmens der EWG, vorsahen, sollten nicht nur die Bürgerinnen und Bürger der Vertragsstaaten profitieren, sondern auch jene in Dänemark, Irland, Italien und dem Vereinigten Königreich, also aller seinerzeitigen EWG-Mitglieder.

Die Regierungen der fünf Schengen-Staaten formulierten das Ziel, den Abbau der Personenkontrollen bis zum Januar 1990 voranzubringen und in der Zwischenzeit Maßnahmen zum Schutz der inneren Sicherheit zur Kompensation des Wegfalls von Kontrollen an den Binnengrenzen zu verhandeln. Diese mündeten in das „Schengener Durchführungsübereinkommen“ (,Schengen II“), das im Dezember 1989 unterzeichnet werden sollte. Dazu aber kam es zunächst nicht: Der Fall der Berliner Mauer am 9. November 1989 und die Öffnung des „Eisernen Vorhangs" schienen neue Unsicherheiten für eine EWG ohne Binnengrenzkontrollen mit sich zu bringen, hatte doch angesichts des „Kalten Krieges“ der Osten Europas in den bisherigen Überlegungen zur Entwicklung eines Schengen-Raums gar keine Rolle gespielt. Für die Bundesrepublik stellte sich außerdem die Frage nach einer Zugehörigkeit des Staatsgebiets der DDR zum Schengen-Raum: Die DDR galt für die Bundesrepublik formell nicht als Ausland, es bestand jedoch die Gefahr, dass die anderen Vertragsstaaten von dort stammende Reisende als Drittstaatsangehörige behandelten.

Mit der absehbaren Vereinigung der beiden deutschen Staaten, die dann im Herbst 1990 erfolgte, erledigte sich dieser Punkt: Bei der Unterzeichnung des Schengener Durchführungsübereinkommens im Juni 1990 wurde die deutschpolnische Grenze als Schengener Außengrenze festgelegt. Der Vertrag trat formell am 1. September 1993 in Kraft, die Grenzkontrollen zwischen den fünf Unterzeichnerstaaten endeten allerdings erst Ende März 1995, also zehn Jahre nach dem Abschluss des Schengener Abkommens.

Als verantwortlich für diese langen Verzögerungen erwiesen sich mehrere Aspekte: In den Verhandlungen Anfang der 1990er Jahre kamen zunehmend stärker die von den nationalen Innenministerien formulierten Sicherheitsinteressen zum Tragen. Demgegenüber verloren jene Ministerien an Gewicht, die für Wirtschaft und für Fragen der europäischen Integration zuständig waren (Baumann 2009; Balch und Geddes 2011, S. 23). In Verbindung damit zu sehen sind die Folgen der vor allem in den späten 1980er Jahren einsetzenden intensiven politischen, öffentlichen und wissenschaftlichen Debatten um die "Globalisierung“ (Bach 2020, S. 139 f.) sowie um die damit in Zusammenhang gebrachte Vorstellung vom Bedeutungsverlust des Nationalstaats und seiner Grenzen: Eine zunehmende 
Verdichtung weitweiter ökonomischer, politischer, sozialer und kultureller Beziehungen sei zu beobachten, die zu vermehrten, von den Nationalstaaten nicht mehr kontrollierbaren Rückkopplungen, Interdependenzen und Abhängigkeiten führe.

Ein Element dieser von einer Kompetenzerosion des Nationalstaates ausgehenden Diagnose, die sich zunehmend verbreitete, bildete die Vorstellung von einer (gewissermaßen unausweichlichen) Zunahme einer kontinentale Grenzen überschreitenden räumlichen Bewegung von Menschen als Begleiterscheinung von Globalisierungsprozessen. In den europäischen Nationalstaaten resultierte aus der Auseinandersetzung um die so vorgestellte Globalisierung ein Bedeutungsgewinn sicherheitspolitischer Argumente: Die als negativ wahrgenommenen Folgen der Globalisierung könnten nur durch eine vermehrte Überwachung und Kontrolle der Grenzen beeinflusst werden. Eile sei angesichts der hohen Geschwindigkeit des Prozesses der Globalisierung dringend geboten, eine intensivierte Zusammenarbeit mit anderen Staaten und deren Sicherheitsbehörden unabdingbar. $^{1}$

Diese Sichtweise gewann auch deshalb an Gewicht, weil sich im Europa der späten 1980er und frühen 1990er Jahre neben der Vorstellung von der „Globalisierung“ auch die Beschreibungskategorie der „Migration“ etablierte und beide Konzepte diskursiv eng miteinander verbunden wurden. Die Herausbildung eines Migrationsdiskurses unter Beteiligung zahlreicher Akteure aus dem politischen, administrativen, zivilgesellschaftlichen und wissenschaftlichen Bereich führte dazu, dass sich die Wahrnehmung räumlicher Bewegungen erheblich verschob: Neu strukturiert bzw. neu zusammengedacht wurden unter dem Begriff der Migration Themenkomplexe, die bis dahin recht unverbundenen gesellschaftlichen Bereichen zuordnet gewesen waren, wie einerseits Flucht/Asyl und andererseits räumliche Bewegungen zur Sicherung von Arbeit und Erwerb, aber auch der demografische und ökonomische Wandel in Europa sowie Bevölkerungswachstum, Armut und wirtschaftliche Entwicklung im Globalen Süden.

Diese weitreichende Verschiebung des Diskurses brachte eine Neuordnung der auf grenzüberschreitende räumliche Bewegungen bezogenen Handlungsfelder und

\footnotetext{
${ }^{1}$ Bemerkenswert erscheint, dass eine solche reflexive Sicht auf die ,Entdeckung“ der Globalisierung bislang kaum anzutreffen ist. Debattiert wird seit vielen Jahren sehr intensiv über den Beginn der Globalisierung, deren Form und Reichweite, selten aber über die Frage, warum das Konzept in den 1980er und frühen 1990er Jahren so rasch rezipiert wurde und welche Folgen die Rezeption für die verschiedensten Zusammenhänge hatte, die mit dem Konzept in Verbindung gebracht wurden. Begrüßenswert sind in diesem Zusammenhang die aktuellen Bemühungen um eine Historisierung der Globalisierung, die auch eine Debatte um die Geschichte des Konzepts Globalisierung und eine kritische Reflexion der Anwendung des Konzepts anregen, hierzu s. vor allem: Wirsching (2020) und Eckel (2020).
} 
deren Bearbeitung (in Verwaltung, im sozialen Sicherungssystem, in Hilfsorganisationen, in zivilgesellschaftlichen Initiativen, in internationalen Agenturen usw.) mit sich. Sie führte auch deshalb zu veränderten Sicht- und Handlungsweisen, weil sie aufgrund der Verknüpfung mit dem Diskurs über „das Globale“ und „die Globalisierung“ Vorstellungen über weltweite räumliche Bewegungen integrierte, insbesondere den Blick auf den Globalen Süden ausrichtete und angesichts des vorgestellten hohen Tempos von Veränderungen durch globale Vernetzungen Gestaltungserfordernisse formulierte, die unter anderem in die Entwicklung von Konzepten eines nur in inter- und supranationaler Abstimmung möglichen „Migrationsmanagements“ mündete. ${ }^{2}$

Die sicherheitspolitisch geprägte Debatte um die Folgen der so verstandenen Globalisierung, die sich durch die Frage nach den Auswirkungen der Öffnung des „Eisernen Vorhangs“ verstärkte, kombinierte sehr eng die Themenfelder Kriminalität und Migration (Huysmans 2000; Baumann 2009): Weitreichende Maßnahmen zur Sicherung der Grenzen seien erforderlich, um gegen die als ansteigend wahrgenommene grenzüberschreitende Kriminalität vorzugehen sowie gegen den „Schmuggel“ oder die „Schleusung“ von Menschen und die „,illegale“, also strafwürdige Migration. Das lässt auch der Wortlaut des „Schengener Grenzkodex" von 2006 deutlich werden, mit dem alle bisherigen Vereinbarungen zu den Grenzkontrollen zusammengefasst worden waren: „Grenzkontrollen sollten zur Bekämpfung der illegalen Zuwanderung und des Menschenhandels sowie zur Vorbeugung jeglicher Bedrohung der inneren Sicherheit, der öffentlichen Ordnung, der öffentlichen Gesundheit und der internationalen Beziehungen der Mitgliedsstaaten beitragen.“3 Zum einen erscheint hier die „Bekämpfung illegaler Zuwanderung" als wichtigster, weil erster Punkt des Aufgabenkanons der Grenzkontrollen. Zum anderen wird sie argumentativ auf eine Stufe gestellt mit der „Bedrohung der inneren Sicherheit, der öffentlichen Ordnung, der öffentlichen Gesundheit und der internationalen Beziehungen der Mitgliedstaaten“ der EU.

Das Schengener Abkommen entfaltete in den 1990er Jahre eine erhebliche Sogwirkung auf andere europäische Staaten: Italien war bereits 1990 beigetreten, Spanien und Portugal folgten 1991, Griechenland 1992. Sie setzten die Regelungen parallel zu den fünf Erstunterzeichnern um. Österreich hatte bereits Mitte der 1980er Jahre Interesse bekundet und schloss sich dem Schengen-Raum

\footnotetext{
${ }^{2}$ Sehr instruktiv, auf den Fall der Schweiz bezogen, mit Perspektiven, die auf andere europäische Gesellschaften übertragbar sind: Espahangizi (2021).

${ }^{3}$ https://eur-lex.europa.eu/legal-content/EN/TXT/PDF/?uri=CELEX:32006R0562\&fro $\mathrm{m}=\mathrm{DE}$ (04.09.2020), s. hierzu auch bereits den Wortlaut der entsprechenden Bestimmungen des Vertrags von Maastricht $1992 \mathrm{im}$ Titel VI, https://eur-lex.europa.eu/legal-content/DE/ TXT/?uri=CELEX:11992M/TXT (18.09.2020).
} 
im Zuge des EU-Beitritts 1995 an. 1996 traten die fünf Staaten der seit 1957 bestehenden „Nordischen Passunion“ (Dänemark, Finnland, Island, Norwegen, Schweden) dem Schengener Abkommen bei, obgleich Norwegen und Island nicht nach der EU-Mitgliedschaft strebten. Auch die Schweiz als Nicht-Mitglied der EU führte Verhandlungen über ein Assoziierungsabkommen, das 2004 geschlossen wurde. Mit dem Amsterdamer Vertrag von 1997 wurde „Schengen“ Teil des EU-Regelungskanons. Großbritannien unterschrieb den Schengener Vertrag nicht. Weil das EU-Mitglied Irland keine Schengen-Grenze zum britischen Nordirland einführen wollte, verzichtete es ebenfalls darauf, Teil des Schengen-Raums zu werden.

Open Access Dieses Kapitel wird unter der Creative Commons Namensnennung 4.0 International Lizenz (http://creativecommons.org/licenses/by/4.0/deed.de) veröffentlicht, welche die Nutzung, Vervielfältigung, Bearbeitung, Verbreitung und Wiedergabe in jeglichem Medium und Format erlaubt, sofern Sie den/die ursprünglichen Autor(en) und die Quelle ordnungsgemäß nennen, einen Link zur Creative Commons Lizenz beifügen und angeben, ob Änderungen vorgenommen wurden.

Die in diesem Kapitel enthaltenen Bilder und sonstiges Drittmaterial unterliegen ebenfalls der genannten Creative Commons Lizenz, sofern sich aus der Abbildungslegende nichts anderes ergibt. Sofern das betreffende Material nicht unter der genannten Creative Commons Lizenz steht und die betreffende Handlung nicht nach gesetzlichen Vorschriften erlaubt ist, ist für die oben aufgeführten Weiterverwendungen des Materials die Einwilligung des jeweiligen Rechteinhabers einzuholen.

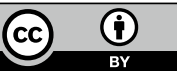

